\title{
Les inconvénients de la maternité au cours d'une carrière médicale
}

\author{
Andrea N. Simpson MD MSc, Maria C. Cusimano MD PhD, Nancy N. Baxter MD PhD
}

Citation : CMAJ 2021 September 20;193:E1465-6. doi : 10.1503/cmaj.211255-f

Voir la version anglaise de l'article ici : www.cmaj.ca/lookup/doi/10.1503/cmaj.211255

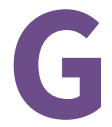

arder un équilibre entre les demandes divergentes de la parentalité et de la carrière en médecine est le défi que vivent plusieurs médecins en exercice et en formation. Retarder le moment d'avoir des enfants pour atteindre certains jalons professionnels importants peut entraîner une augmentation du taux d'infertilité et de complications pendant la grossesse chez les femmes médecins ${ }^{1}$. Par ailleurs, avoir des enfants de façon précoce, au cours de la formation médicale, est tout un défi et peut influencer les décisions professionnelles d'une stagiaire. Des présomptions portant sur la manière dont les priorités d'une femme médecin vont changer lorsqu'elle aura des enfants peuvent entraîner un nombre moindre d'occasions professionnelles et encourager à se spécialiser dans des domaines qui sont mieux adaptés à la vie familiale ${ }^{2}$. Et pourtant, le fardeau de performer dans une profession qui a historiquement exclu les femmes et qui n'a reconnu que tout récemment l'importance du bien-être du médecin et de la culture de l'épanouissement personnel pèse sur les épaules des femmes médecins. Malgré les récentes avancées dans la promotion du bien-être des médecins, la parentalité, particulièrement la maternité, est perçue comme une perturbation pendant la formation médicale et au-delà; dans la profession médicale, il ne semble pas exister de "bon " moment pour avoir des enfants. Notre profession fait l'objet de travaux de recherche où l'on mentionne que jusqu'à une femme médecin sur quatre rapporte un diagnostic d'infertilité ${ }^{1}$. Cela devrait susciter un appel à l'action pour des changements systémiques en médecine, particulièrement pour les parcours de formation médicale qui sont prolongés.

Les femmes médecins font couramment état d'un retard dans la prise de décisions en lien avec leur vie personnelle en raison de leurs objectifs professionnels ${ }^{3,4}$. Celles qui font le choix de poursuivre une grossesse pendant leur formation mentionnent souvent être l'objet de discrimination maternelle, entraînant des conséquences professionnelles négatives ${ }^{5}$. La discrimination maternelle consiste en une discrimination fondée sur le sexe visant spécifiquement le statut de la maternité; elle peut être vécue comme un manque de soutien pendant et après la grossesse, un manque d'accommodements pour répondre

\section{Points clés}

- Une grossesse pendant la formation médicale et, par la suite, au cours de la carrière médicale, est souvent encore perçue comme une perturbation ou un fardeau, malgré l'importance grandissante accordée au bien-être des médecins.

- En raison de la stigmatisation et des obstacles systémiques, plusieurs médecins commencent à procréer à un âge plus avancé que leurs homologues qui ne sont pas médecins, ce qui peut s'accompagner d'un risque accru d'infertilité, de fausses couches et d'autres complications liées à la grossesse.

- Les femmes médecins retardent la procréation sur une plus longue période, ce qui pourrait être associé aux parcours de formation plus étendus.

- Les interventions ayant pour but d'atténuer la discrimination contre les médecins en âge de procréer peuvent comprendre une augmentation du nombre de postes de résidence afin d'assurer une flexibilité des horaires, l'intégration de l'enseignement de la santé reproductive destinée aux médecins dans le curriculum médical au début de la formation et l'encouragement de regroupements d'alliés au sein de la profession pour réduire la stigmatisation.

aux défis posés par les soins aux enfants, des remarques désobligeantes en lien avec la grossesse et la maternité et la mise à l'écart de possibilités de carrières ${ }^{5}$. Sur la base d'aspirations familiales présumées, les femmes qui étudient la médecine sont plus susceptibles de se faire déconseiller de poursuivre leurs études dans des domaines de spécialisation comme la chirurgie en raison de leur sexe ${ }^{6}$. Lorsque ces facteurs sont pris en considération, il n'est pas surprenant de constater que plusieurs femmes médecins choisissent de retarder le moment d'avoir des enfants bien que le coût personnel peut souvent être élevé considérant l'augmentation de l'incidence des fausses couches, de l'infertilité et des complications vécues pendant la grossesse en raison de l'âge maternel avancé ${ }^{1,7}$.

La formation médicale coïncide souvent avec l'âge biologique optimal pour avoir des enfants. Les femmes médecins doivent souvent prendre des décisions difficiles en lien 
avec le choix d'avoir ou non des enfants, à quel moment, combien, tout en essayant d'atteindre leurs objectifs professionnels. Nous avons récemment cartographié la trajectoire de l'accouchement chez les femmes médecins dans le cadre d'une étude populationnelle faisant appel à des données ontariennes, au Canada ${ }^{8,9}$. Nous avons découvert que cette culture de report se manifeste de façon précoce et persiste tout au long de la formation postdoctorale. Nous avons trouvé un taux d'accouchement significativement moins élevé chez les femmes médecins que chez les femmes de la population générale et un taux accéléré de naissances uniquement à des âges reproductifs avancés, suggérant que les femmes médecins semblent retarder leur grossesse jusqu'au moment où leur formation est complétée, les médecins spécialistes retardant leur grossesse davantage que les médecins de famille.

En tant que profession, nous devons réfléchir de façon plus ambitieuse à la manière dont nous pouvons soutenir les médecins qui souhaitent avoir des enfants, peu importe le stade de leur carrière. L'offre de politiques de congés parentaux et de soutien financier accru, de paramètres pour les gardes de nuit en cours de grossesse pour les stagiaires postdoctoraux et des accommodements pratiques comme l'accessibilité à des ressources en allaitement au sein des hôpitaux ont constitué des interventions substantielles et positives au niveau du système et des établissements ${ }^{10}$. Par contre, malgré ces changements ${ }^{10}$, nous avons découvert que la durée du report de la grossesse est en croissance - les femmes médecins nées entre 1985 et 1994 ayant une incidence cumulée d'accouchements à des âges comparables inférieure à celles nées entre 1976 et $1984^{8}$. Bien que le prolongement des trajectoires de formation ou l'accroissement des difficultés à obtenir une carrière professionnelle stable soient des facteurs déterminants, ces découvertes sont aussi le reflet de suppositions sous-jacentes qui alimentent la discrimination maternelle en médecine. Les médecins qui s'absentent pour un congé parental ou de grossesse décrivent souvent ce temps passé à l'écart comme un «fardeau »-comme le considèrent aussi leurs pairs - qui augmente la charge de travail pour les autres collègues 5 . Les étudiants en médecine et les résidents n'ont souvent pas les moyens de payer pour des options flexibles de garde d'enfants. Les femmes médecins mentionnent une discrimination maternelle omniprésente ${ }^{5}$, accompagnée d'une diminution des occasions professionnelles après avoir eu des enfants ${ }^{2}$.

On doit accorder plus d'importance à l'enseignement de la santé reproductive destiné aux médecins, particulièrement tôt dans la formation médicale, afin de s'assurer que les médecins puissent prendre des décisions éclairées en lien avec leur planification familiale. Bien que la préservation de la fertilité au moyen de la congélation optionnelle d'ovules soit souvent présentée comme une façon de contourner les demandes de la formation médicale ${ }^{4}$, ce n'est pas une solution à toute épreuve et certainement pas une solution idéale à l'enjeu plus étendu du report de la grossesse; ce ne devrait pas être la seule option. Nos milieux de formation doivent s'adapter afin que chaque domaine de spécialisation soit solidaire des personnes qui désirent devenir parents.
Bien que nous nous soyons concentrées principalement sur les enjeux en lien avec la maternité dans le milieu médical, l'atteinte de l'équilibre entre la famille et une carrière en médecine ne devrait pas être envisagée comme un problème qui touche uniquement les femmes - les points de vue des hommes et des médecins présentant une identité sexuelle diversifiée sont importants et ont peu fait l'objet de recherches. Afin de promouvoir le soutien d'alliés, il est important de mobiliser des médecins de tous les sexes, particulièrement ceux occupant des postes de direction, afin de percevoir le congé parental comme une clé de la santé des médecins et de leurs enfants plutôt qu'un fardeau à poser sur les épaules des collègues. À plus large échelle, augmenter le financement des postes de résidence afin d'assurer un nombre adéquat de stagiaires pour répondre aux besoins des services en fonction des congés parentaux pourrait favoriser l'acceptation des grossesses et des congés parentaux par les autres stagiaires et améliorer la flexibilité des horaires. Les répercussions de la discrimination contre les médecins qui voudraient être enceintes sont substantielles, allant jusqu'à la décision de certaines femmes médecins de changer de lieu de pratique en raison d'un environnement de travail toxique ou quittant tout simplement la profession ${ }^{5}$. Un milieu qui force un médecin à quitter la pratique de la médecine après avoir investi des années de formation dans cette profession est une tragédie évitable pour la personne, la communauté médicale et les patients qui sont touchés. Encourager un changement positif systémique de la médecine pour soutenir les médecins qui ont des enfants, peu importe le stade de leur formation, favorisera le bien-être et la satisfaction professionnelle à long terme.

\section{Références}

1. Stentz NC, Griffith KA, Perkins E, et al. Fertility and childbearing among American female physicians. J Womens Health (Larchmt) 2016;25:1059-65.

2. Adesoye T, Mangurian C, Choo EK, et al.; Physician Moms Group Study Group. Perceived discrimination experienced by physician mothers and desired workplace changes: a cross-sectional survey. JAMA Intern Med 2017;177:1033-6.

3. Bering J, Pflibsen L, Eno C, et al. Deferred personal life decisions of women physicians. J Womens Health (Larchmt) 2018;27:584-9.

4. Nasab S, Shah JS, Nurudeen K, et al. Physicians' attitudes towards using elective oocyte cryopreservation to accommodate the demands of their career. J Assist Reprod Genet 2019;36:1935-47.

5. Halley MC, Rustagi AS, Torres JS, et al. Physician mothers' experience of workplace discrimination: a qualitative analysis. BMJ 2018;363:k4926.

6. Giantini Larsen AM, Pories S, Parangi S, et al. Barriers to pursuing a career in surgery: an institutional survey of Harvard Medical School students. Ann Surg 2021;273:1120-6.

7. Rangel EL, Castillo-Angeles M, Easter SR, et al. Incidence of infertility and pregnancy complications in US female surgeons. JAMA Surg 2021 July 28 [cyberpublication avant impression]. doi: 10.1001/jamasurg.2021.3301.

8. Cusimano MC, Baxter NN, Sutradhar R, et al. Delay of pregnancy among physicians vs nonphysicians. JAMA Intern Med 2021;181:905-12.

9. Cusimano MCBN, Sutradhar R, Ray J, et al. Reproductive patterns, pregnancy outcomes and parental leave practices of women physicians in Ontario, Canada: the Dr Mom Cohort Study protocol. BMJ Open 2020; 10:e041281.

10. Pregnancy and parental leave. Toronto: Professional Association of Residents of Ontario (PARO). Accessible ici : https://myparo.ca/your-contract/\#pregnancy -and-parental-leave (consulté le 16 juin 2021). 
Intérêts concurrents : Aucun déclaré.

Cet article a été révisé par des pairs.

Affiliations : Département d'obstétrique et de gynécologie (Simpson, Cusimano), Université de Toronto; Institut du savoir Li Ka Shing (Simpson, Baxter), Hôpital St. Michael; Service d'obstétrique et de gynécologie (Simpson, Cusimano, Baxter), Hôpital St. Michael/Réseau hospitalier Unity Health de Toronto; ICES Central (Simpson), Toronto, Ont.; École de santé des populations et de santé mondiale de Melbourne (Baxter), Université de Melbourne, Melbourne, Victoria, Australie.
Collaborateurs : Toutes les auteures ont contribué à l'élaboration et à la conception du travail. Andrea Simpson a produit l'ébauche du manuscrit. Toutes les auteures ont révisé de façon critique son contenu intellectuel important, ont donné leur approbation finale pour la version destinée à être publiée et endossent l'entière responsabilité de tous les aspects du travail.

Soutien financier : Parmi les études citées, celles réalisées par les auteures ${ }^{8,9}$ ont obtenu le soutien financier de la fondation Physicians' Services Incorporated.
Propriété intellectuelle du contenu : Il s'agit d'un article en libre accès distribué conformément aux modalités de la licence Creative Commons Attribution (CC BY-NC-ND 4.0), qui permet l'utilisation, la diffusion et la reproduction de tout médium à la condition que la publication originale soit adéquatement citée, que l'utilisation se fasse à des fins non commerciales (c.-à-d., recherche ou éducation) et qu'aucune modification ni adaptation n'y soit apportée. Voir : https://creativecommons.org/ licenses/by-nc-nd/4.0/deed.fr.

Correspondance : Andrea Simpson, andrea.simpson@unityhealth.to 